\title{
Compassion fatigue among oncologists: the role of grief, sense of failure, and exposure to suffering and death
}

\author{
Rony Laor-Maayany ${ }^{1} \cdot$ Gil Goldzweig $^{1} \cdot$ Ilanit Hasson-Ohayon ${ }^{2} \cdot$ Gil Bar-Sela $^{3} \cdot$ Adi Engler-Gross $^{1} \cdot$ Michal Braun $^{1,4}$ (1)
}

Received: 2 May 2019 / Accepted: 24 July 2019 /Published online: 8 August 2019

(C) Springer-Verlag GmbH Germany, part of Springer Nature 2019

\begin{abstract}
Purpose Oncologists cope with unique work characteristics that increase their risk of developing compassion fatigue - that is, burnout and secondary traumatic stress - and can result in reduced capacity and interest in being empathetic to the suffering of others (Stamm B. The concise ProQOL manual, 2010). At the same time, oncologists can experience compassion satisfactionthat is, the positive aspects of caring. This study explored the associations of compassion fatigue and compassion satisfaction with oncologists' grief and sense of failure beyond their reported exposure to suffering and death.

Methods Seventy-four oncologists completed self-administered questionnaires examining compassion fatigue, compassion satisfaction, grief, exposure to suffering and death, and sense of failure.

Results The oncologists reported that they face the loss of approximately $50 \%$ of their patients, and that their patients suffer from profound emotional and physical pain. High levels of compassion fatigue and grief, and moderate levels of sense of failure, were reported. Findings showed a lack of association between exposure to suffering and death and compassion fatigue and satisfaction. However, grief and sense of failure were found to predict both aspects of compassion fatigue: secondary traumatic stress $(p<0.001, p<0.003$, respectively) and burnout ( $p<0.002, p<0.025$, respectively).

Conclusions These results highlight the importance of the oncologists' subjective experiences of grief and sense of failure, beyond their reports of exposure to suffering and death, in terms of their levels of compassion fatigue. Implications of these findings include the need to develop interventions for oncologists that will allow them to acknowledge, process, and overcome negative experiences of failure and grief.
\end{abstract}

Keywords Compassion fatigue $\cdot$ Burnout $\cdot$ Oncology $\cdot$ Grief $\cdot$ Sense of failure

\section{Introduction}

Cancer is one of the leading causes, worldwide, of mortality [1]. As such, oncologists have much exposure to suffering, dying, and death. On the other hand, increased survival rates have led to long-lasting and intense oncologist-patient relationships [2]. This combination may put oncologists at increased risk for developing compassion fatigue.

Michal Braun

bmichalpsy@gmail.com

1 The Academic College of Tel Aviv-Yaffo, Tel Aviv, Israel

2 Department of Psychology, Bar-Ilan University, Ramat Gan, Israel

3 Emek Medical Center, Afula, Israel

4 The Talya Center for Young Women with Breast Cancer, Shaare Zedek Medical Center, Jerusalem, Israel
Compassion fatigue is defined as a state of tension, which can develop among professional caregivers in response to witnessing the suffering of others. It occurs when stress accumulates due to the constant need to relieve others' suffering; its consequences include the caregiver's reduced capacity and interest in being empathetic to suffering others, and in his/her reduced personal and professional well-being [3].

Stamm [4] suggested a theoretical model conceptualizing compassion fatigue as a negative aspect of working in the helping professions and compassion satisfaction as a positive aspect. Compassion fatigue is composed of burnout and secondary traumatic stress (STS), both of which are regarded as negative implications of the work. Burnout usually develops in response to work environment characteristics (e.g., workload, nonsupportive work environment) and includes feelings of exhaustion, frustration, anger, and depression. Secondary traumatic stress develops in response to exposure to patients' traumatic events, and its symptoms include intrusive thoughts, 
avoidant behavior, and high levels of tension $[4,5]$. Compassion satisfaction is regarded as embodying the positive feelings that professionals might derive from their practice, such as the pleasure of helping others and the feeling of success in helping others $[4,5]$. As compassion fatigue and compassion satisfaction can coexist [4], some researchers view compassion satisfaction as a protective factor against the development of compassion fatigue [6].

The phenomenon of compassion fatigue is documented among physicians in general $[7,8]$ and specifically among oncologists [9], with $47 \%$ of US oncologists reporting compassion fatigue symptoms [10]. Compassion fatigue has also been reported by other oncology health care professionals, such as nurses and social workers $[11,12]$. The implications of compassion fatigue for the personal and professional wellbeing of professional caregivers are documented in the literature, with studies showing burnout's association with patient safety, professionalism, and patient satisfaction (see Panagioti et al. recent meta-analysis [7]). The phenomenon of distancing or withdrawing from patients as the patient moves closer to death (e.g., fewer bedside visits) was also documented with regard to compassion fatigue among oncologists [13].

Exposure to suffering and death does not necessarily lead to compassion fatigue [4]. It seems, instead, that the oncologist's internal emotional processes may affect compassion fatigue and compassion satisfaction. Thus, in the current study, we examined whether grief and a sense of failure regarding the oncologist's own professional work affected compassion fatigue and compassion satisfaction beyond his/her reported exposure to death and suffering.

Grief is a well-known response to the death of a beloved person, usually a family member or a good friend. Nevertheless, oncologists may also experience grief over the deaths of their patients. Giddings [14] wrote that grief is usually experienced by oncologists in response to the loss of a patient who has touched them in some particular way or who has reminded them of the reasons they chose to go into their profession. Granek and colleagues $[9,13,15-17]$ conducted an in-depth qualitative investigations of oncologists' grief and pointed to its uniqueness in that it derives, at least in part, from a sense of responsibility for the patient's life. Oncologists' grief includes, besides sorrow, feelings of powerlessness, self-doubt, and guilt [13]. Interestingly, no correlation was found between the time that had passed since the patient's death and the duration of the oncologist's grief, which might last only a few hours or, alternatively, never entirely disappear [16]. It has been suggested that when oncologists' grief is not treated, they may lose the capacity to be compassionate and dedicated to their patients [18], potentially an expression of compassion fatigue. Moreover, grief may cause the oncologist to alter treatment decisions or withdraw from patients and their families [13]. Despite this considerable potential impact on oncologists at both the personal and professional levels, oncologists' grief has not been, to the best of our knowledge, quantitatively examined.

Like grief, sense of failure is also a widely recognized experience; it arises when unwanted outcomes take place. For oncologists, a sense of failure can arise when a treatment plan fails or a patient's dies; it can also emerge when oncologists hold on to unrealistic expectations of themselves [19] or fail to produce a "miracle" [18]. A sense of failure can have a deleterious influence on patient care, leading for instance to the use of additional aggressive treatments when a palliative approach would be more appropriate [18]. Sense of failure among physicians, and among oncologists in particular, has rarely been documented. To the best of our knowledge, there is only one survey that directly examined sense of failure among oncologists [20]. In this survey, out of the 598 American oncologists who responded, 56\% reported experiencing burnout symptoms. Of them, $56 \%$ reported experiencing a sense of failure.

\section{Hypotheses}

The current study explored the predictors of compassion fatigue (i.e., burnout and STS) and compassion satisfaction. Specifically, it assessed whether grief over patients and sense of failure affected these outcomes beyond the oncologists' reported exposure to death and suffering. We hypothesize that these internal emotional processes will positively associated with compassion fatigue. In addition, we examined the association of grief over patients and sense of failure to compassion satisfaction, while we had no directional hypotheses due to lack of literature on the topic.

\section{Methods}

\section{Participants and procedure}

Recruitment for this study took place at the 2017 Annual Meeting of the Israel Society for Clinical Oncology and Radiation Therapy (ISCORT) and at the 2017 Spring Meeting of the Israel Society of Hematology and Transfusion Medicine, resulting in the participation of 92 out of a total of approximately 270 Israeli oncologists. The participants signed an informed consent form and filled out the questionnaires via Qualtrics ${ }^{\circledR}$ using their smartphones or computers. To begin the study, participants either scanned a $\mathrm{QR}$ code or received a link in a text message from the research team. In appreciation for their participation, the participants took part in a lottery. The study received ethical approval from the Academic College of Tel Aviv-Yaffo Ethics Board (approval 2018001). 


\section{Measures}

The formal Hebrew-language version of the Professional Quality of Life Questionnaire-ProQOL (version 5) [4] was used to assess compassion fatigue and compassion satisfaction. It consists of 30 items, with a 6-point response range scale for each item. The questionnaire includes three subscales: STS (e.g., "I feel depressed because of the traumatic experiences of the people I help"), burnout (e.g., "I feel trapped by my job as a helper"), and compassion satisfaction (e.g., "I get satisfaction from being able to help people"). In the present study, Cronbach alpha coefficients were adequate (STS: $\alpha=0.88$, burnout: $\alpha=0.70$, compassion satisfaction: $\alpha=0.79$ ).

The Hebrew-language version [21] of the Texas Revised Inventory of Grief-TRIG-Present Scale [22] was administered. This measure consists of 13 items, with a 5-point response range scale for each item, assessing participants' current grief feelings about past loss. In the original questionnaire, a higher score indicated a lower level of grief. During the data analysis phase of our study, we reversed the scale, with higher scores indicating higher levels of grief, so that all of the variables in the model would have the same directionality. In the present study, the Cronbach alpha coefficient was 0.89 .

Sense of failure regarding the participant's professional work was examined using a single item, as was done previously by Whippen and Canellos [20] in their research on oncologists' burnout. The answers varied from very little (1) to extremely (5).

Exposure to suffering and death was measured using three items in which the participants were asked to estimate the percentages (from 0 to $100 \%$ ) of their patients who died from the disease, as well as the percentages of their patients who suffer from moderate to severe physical and/or emotional pain during a significant amount of the time.

The sociodemographic questionnaire consisted of questions regarding personal information (e.g., gender and age) and questions regarding work (e.g., seniority and place of work).

\section{Statistical analysis}

Data were analyzed using the Statistical Package for the Social Sciences, version 25 (SPSS, Chicago, IL). Statistical tests were two-tailed with $\alpha$ set at 0.05 . Descriptive statistics were calculated to provide information about participants' characteristics and the prevalence of the main variables of the study. In order to test our main hypotheses, we used a hierarchical regression model to test both main and interaction effects.

Power considerations In order to determine the sample size for the research, we used calculations based on Cohen's definitions for effect size and power calculations [23]. Calculations were made using "G*Power" computer software [24]. We calculated power for $R$ square increase in hierarchical regression with 2 blocks: the first block included the independent variables of exposure (e.g., exposure to death, physical and emotional pain), and the second block included the oncologist's emotional internal processes variables (grief and sense of failure). The sample size needed in order to achieve power (1-beta) of $90 \%$, given a priori alpha error probability $=0.05$ and expected medium effect size $\left(F^{2}=0.15\right)$ is 88 .

\section{Results}

\section{Sociodemographic variables}

Seventy-four oncologists completed the questionnaires. The average participant age was 45.1 years $(\mathrm{SD}=11.41$ years, range 29-75 years); $56.2 \%$ of the participants were female, 95.9\% of the participants were married or living with a partner, and $84.7 \%$ of the participants had children.

The majority of the participants worked as medical oncologists (82.4\%). Most of them practiced in hospitals (94.6\%) and had full-time positions (91.9\%). Of the participants, $68.9 \%$ were senior oncologists and $31.1 \%$ were residents, with an average of 13.9 years since the time of their training ( $\mathrm{SD}=11.34$, range $1-42$ years). Participants had various specialties: breast and gynecology (27.9\%), lung (14.7\%), hematology $(22.1 \%)$, or others $(23.5 \%)$. At the time of the study, $11.8 \%$ did not specialize. Oncologists and hematooncologists showed no significant differences across any of the study's measurements.

\section{Exposure to suffering and death}

High levels of exposure to suffering and death were reported. The participants estimated that on average $41.74 \%$ ( $\mathrm{SD}=$ 23.62) of their patients died of the cancer. As can be seen in Fig. 1a, only a small percentage of oncologists reported not being exposed to patients' deaths, whereas about $40 \%$ estimated that $29-50 \%$ of their patients died of the cancer, and $10 \%$ estimated that between 73 and $100 \%$ of their patients died of the cancer.

In regard to exposure to suffering, there were higher levels reported of exposure to emotional suffering than of exposure to physical suffering $\left(t_{73}=8.63, p<0.000\right)$. The participants estimated that on average, $32.58 \%(\mathrm{SD}=21.06 \%)$ of their patients suffer from medium to severe levels of physical pain during a significant amount of the time (see Fig. 1b), whereas $54.45 \%$ ( $\mathrm{SD}=24.18 \%$ ) of their patients suffer from emotional pain during a significant amount of the time. Moreover, extremely high levels of exposure to emotional suffering were reported by $25 \%$ of the participants, who estimated that more than $71 \%$ of their patients suffer from medium to high levels 

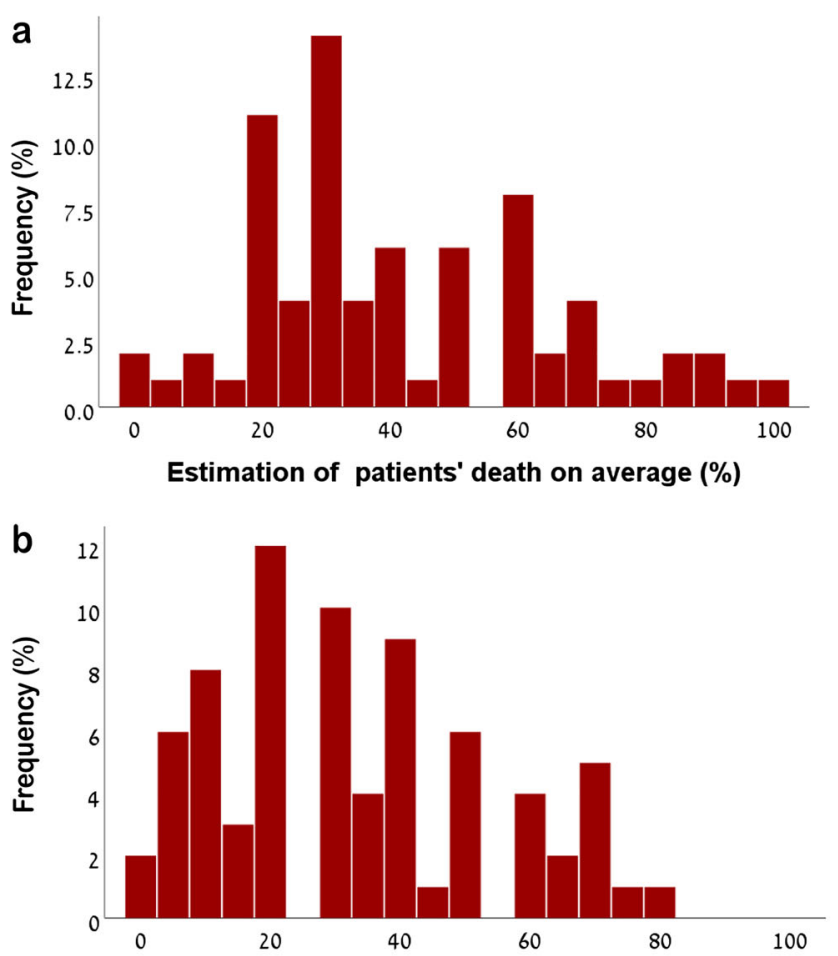

Estimation of patients suffering from physical pain (\%)

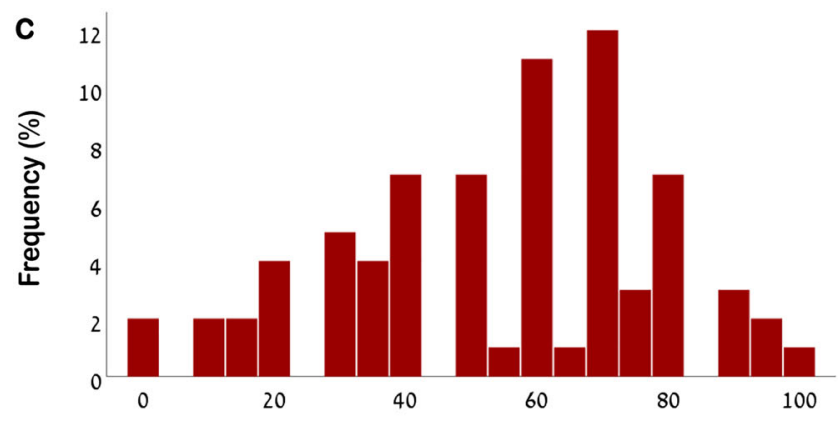

Estimation of patients suffering from emotional pain (\%)

Fig. 1 Exposure to suffering and death. The participants estimated that on average $41.74 \%(\mathrm{SD}=23.62)$ of their patients died from the disease $(\mathbf{a})$, $32.58 \%(\mathrm{SD}=21.06)$ of their patients suffer from medium to severe levels of physical pain $(\mathbf{b})$, and that $54.45 \%(\mathrm{SD}=24.18)$ of the patients suffer from emotional pain during a significant amount of the time (c)

of emotional pain during a significant amount of the time (see Fig. 1c).

\section{Secondary traumatic stress, burnout, and compassion satisfaction}

Compared with published norms by Stamm [5], STS levels were found to be high, with an average score of 17.18 (SD = 7.02 , range $=3-39$ ). Similarly, burnout levels were also high, with an average score of $26.74(\mathrm{SD}=6.76$, range $=4-41)$. Compassion satisfaction levels were found to be moderate, with an average of $36.86(\mathrm{SD}=7.17$, range $=14-50)$.

\section{Grief and failure}

Participants reported a mild sense of failure, with an average score of 2.19 ( $\mathrm{SD}=0.87$, range $=1-5$ ). Grief levels were relatively high, but did not reach the cut point for pathological grief [22], with an average of 26.21 ( $\mathrm{SD}=8.96$, range $=13-50)$.

In summary, the majority of the oncologists reported that they are exposed to high levels of patients' physical and emotional suffering, as well as to high rates of patients' death, during their daily work.

\section{Correlations analyses}

Pearson's correlations between the study's main variables were calculated (see Table 1). None of the correlations between the exposure variables and STS, burnout, and compassion satisfaction was found to be significant. Grief was highly positively correlated with STS and moderately positively correlated with burnout. Sense of failure was moderately positively correlated with STS and burnout and moderately negatively correlated with compassion satisfaction. Grief and sense of failure were not significantly correlated.

As could be expected, we found a high positive correlation between STS and burnout. Additionally, we found a negative moderate correlation between burnout and compassion satisfaction, and a low negative correlation between STS and compassion satisfaction.

\section{Regression model predicting compassion fatigue and compassion satisfaction}

Two-step hierarchical multiple regressions for each of the predicted variables-STS, burnout, and compassion satisfaction - were conducted. In all of the hierarchical multiple regressions, the first block included the independent variables of exposure (e.g., exposure to death, physical and emotional pain), and the second block included the oncologist's emotional internal processes variables (grief and sense of failure). No associations were found between the demographic variables and the dependent variables; therefore, they were not entered into the regressions. All variables were centered prior to the analysis.

As can be seen from Table 2, the regression models predicting STS (adjusted $R^{2} 0.424, F_{(5,68)} 11.741, p<0.001$ ) and burnout (adjusted $R^{2} 0.212, F_{(5,68)} 4.922, p<0.002$ ) were found to be significant, but the regression model predicting compassion satisfaction (adjusted $R^{2} 0.067, F_{(5,68)} 2.049, p=$ 0.083 ) was not.

In all of the regression models, the first step (exposure to death and suffering as predictors) was not significant. In the regressions, when STS and burnout were the predicted variables, the second step was significant. Grief and sense of failure were the only final significant predictors of STS (grief: 
Table 1 Pearson's correlations between the main model variables

\begin{tabular}{|c|c|c|c|c|c|c|c|c|}
\hline & $\begin{array}{l}\text { Exposure } \\
\text { to death }\end{array}$ & $\begin{array}{l}\text { Exposure to } \\
\text { physical } \\
\text { pain }\end{array}$ & $\begin{array}{l}\text { Exposure to } \\
\text { emotional } \\
\text { pain }\end{array}$ & $\begin{array}{l}\text { Sense of } \\
\text { failure }\end{array}$ & Grief & $\begin{array}{l}\text { Secondary } \\
\text { traumatic } \\
\text { stress }\end{array}$ & Burnout & $\begin{array}{l}\text { Compassion } \\
\text { Satisfaction }\end{array}$ \\
\hline Exposure to death & 1 & $0.251 *$ & $0.424 * *$ & -0.082 & 0.026 & 0.048 & 0.114 & -0.076 \\
\hline Exposure to physical pain & & 1 & $0.543 * *$ & $0.281 *$ & -0.066 & 0.056 & 0.098 & -0.097 \\
\hline Exposure to emotional pain & & & 1 & 0.161 & 0.020 & 0.004 & 0.220 & -0.122 \\
\hline Sense of failure & & & & 1 & 0.107 & $0.357 * *$ & $0.301 * *$ & $-0.340^{* *}$ \\
\hline Grief & & & & & 1 & $0.603 * *$ & $0.404 * *$ & -0.086 \\
\hline Secondary traumatic stress & & & & & & 1 & $0.638 * *$ & $-0.261 *$ \\
\hline Burnout & & & & & & & 1 & $-0.521 * *$ \\
\hline Compassion satisfaction & & & & & & & & 1 \\
\hline
\end{tabular}

*Correlation is significant at the 0.05 level (2-tailed)

$* *$ Correlation is significant at the 0.01 level (2-tailed)

beta $0.573, p<0.001$; sense of failure: beta $0.31, p<0.003$ ) and burnout (grief: beta $0.367, p<0.002$; sense of failure: beta $0.257, p<0.025$ ). The only significant inverse predictor of compassion satisfaction was sense of failure (beta -0.349 , $p<0.006)$.

\section{Discussion}

The current study addressed the predictors of oncologists' compassion fatigue and satisfaction. Controlling for their reported exposure to death and suffering, we sought to test whether their internal emotional processes of grief and sense of failure affected their compassion. Findings showed no associations between exposure to suffering and death, and compassion fatigue and satisfaction. Findings also revealed the significant role played by grief and sense of failure as predictors for both aspects of compassion fatigue: STS and burnout. Notably, the lack of association between exposure variables and STS, burnout, and compassion satisfaction runs contrary to the prevalent theory and to prior empirical findings $[3,25]$.

One possible explanation for the lack of association between exposure to death and suffering, and compassion fatigue and satisfaction, may be the unique characteristics of our participants, who are highly exposed to patients' suffering and deaths; that is, oncologists' high levels of exposure may create a ceiling effect that masks the association between the two. In other words, most oncologists are exposed to a significant and high amount of patient suffering and death; therefore, the variance between them does not result in implications for their compassion fatigue.

Another possible explanation for the lack of association might be that the exposure, in and of itself, is not significant in the development of compassion fatigue; rather, it may be that the subjective experience elicited by the exposure is the significant aspect. As we suggested, oncologists may need to be "touched" by the suffering and deaths they are exposed to in order to develop compassion fatigue symptoms. In our study, these subjective experiences were represented by the oncologists' grief and sense of failure. The greater significance of subjective aspects over objective ones in the development of emotional distress has been revealed in previous studies among different samples [26-28]. For example, Braun and colleagues showed the preeminence of subjective aspects, over objective ones, of caregiving burden in predicting caregivers' depression among caregiver spouses of patients with advanced cancer [26].

Compassion fatigue, a known risk factor among professional caregivers, is considered to be a result of secondary

Table $2 R^{2}$ change for compassion fatigue, burnout, and compassion satisfaction

\begin{tabular}{|c|c|c|c|c|c|c|}
\hline & \multicolumn{2}{|c|}{ Secondary traumatic stress } & \multicolumn{2}{|l|}{ Burnout } & \multicolumn{2}{|c|}{ Compassion satisfaction } \\
\hline & Adjusted $R^{2}$ & $R^{2}$ change & Adjusted $R^{2}$ & $R^{2}$ change & Adjusted $R^{2}$ & $R^{2}$ change \\
\hline Step 1: exposure variables & -0.036 & 0.007 & 0.009 & 0.049 & -0.025 & 0.017 \\
\hline Step 2: grief and sense of failure & 0.424 & $0.457^{* *}$ & 0.212 & $0.216^{* * *}$ & 0.067 & $0.114^{*}$ \\
\hline
\end{tabular}

*Correlation is significant at the 0.05 level (2-tailed)

**Correlation is significant at the 0.01 level (2-tailed) 
exposure to others' traumatic experiences [3]. As reported herein, oncologists are indeed highly exposed to the suffering and deaths of their patients. Unlike other professional caregivers (e.g., psychotherapists), oncologists do not learn about their patients' traumas merely through patients' reports and stories; rather, they witness their patients' suffering firsthand. Moreover, oncologists often perceive themselves as being responsible for the suffering experienced by their patients, and even for their deaths [18]. Therefore, the repeated experiences of grief and sense of failure can be regarded as primary traumas experienced by the oncologists themselves $[15,17$, 29], rather than as secondary exposure to traumatic experiences. It is possible that oncologists develop compassion fatigue in response to their primary exposure to the trauma of repeated loss and perceived failure, and not only in response to their secondary exposure to suffering and death.

An additional finding of the current study was the low to moderate negative correlations between compassion fatigue aspects and compassion satisfaction, indicating that the two constructs are not "two sides of the same coin" but two separate constructs that can coexist. In other words, the fact that the oncologists reported high levels of compassion fatigue did not prevent them from experiencing compassion satisfaction. Interestingly, it seems that compassion satisfaction was more negatively associated with the burnout aspect of compassion fatigue than with the STS. As burnout is usually related to work environment [4], it is possible that this factor could be modified in order to increase oncologists' compassion satisfaction.

It is not surprising that our model was nonsignificant for compassion satisfaction. Given that compassion satisfaction is the expression of the positive feelings one derives from helping others, we expected it to be more associated with resilience factors and coping mechanisms than with negative feelings such as grief and sense of failure $[30,31]$. Nevertheless, we found a negative association between compassion satisfaction and sense of failure. This association highlights the importance of focusing on sense of failure in future interventions designed to improve oncologists' professional quality of life.

Notwithstanding there are limitations to the study. First, the cross-sectional nature of the study does not allow causal inferences nor did it allow us to trace the dynamic changes of compassion fatigue: a construct which has been found to decrease as professional experience increases [20,32]. However, we found no association between oncologist seniority and levels of compassion fatigue or satisfaction. Second, the sample's homogeneity in terms of certain sociodemographic characteristics limited our ability to look for associations between these characteristics and the development of compassion fatigue.

Considering these limitations in mind, the findings of the current study suggest that psychological interventions with oncologists at the level of their subjective experience might be beneficial. Although it is not possible to change oncologists' exposure to profound levels of suffering and death, it is possible to change their perceptions regarding their role and their experience. Specifically, providing oncologists with an opportunity to process and reflect on their role and on their losses might ease their distress and, accordingly, improve their ability to be available to their patients.

Acknowledgments We acknowledge the Israel Cancer Association for supporting and funding this study (Grant number 20180116).

Data availability The data that support the findings of this study are available from the corresponding author upon reasonable request.

Compliance with ethical standards The study received ethical approval from the Academic College of Tel Aviv-Yaffo Ethics Board (approval 2018001).

Conflict of interest Dr. Braun, Mrs. Laor-Maayany, and Mrs. EnglerGross report grants from the Israel Cancer Association, during the conduct of the study; Prof. Goldzweig, Prof. Hasson-Ohayon, and Dr. BarSela have nothing to disclose.

\section{References}

1. Plummer M, de Martel C, Vignat J, Ferlay J, Bray F, Franceschi S (2016) Global burden of cancers attributable to infections in 2012: a synthetic analysis. Lancet Glob Heal 4:e609-e616. https://doi.org/ 10.1016/S2214-109X(16)30143-7

2. Eide H, Graugaard P, Holgersen K, Finset A (2003) Physician communication in different phases of a consultation at an oncology outpatient clinic related to patient satisfaction. 51:259-266

3. Figley CR (2002) Compassion fatigue: psychotherapists' chronic lack of self care. J Clin Psychol 58:1433-1441. https://doi.org/10. 1002/jclp. 10090

4. Stamm B (2010) The concise ProQOL manual. Pocatello, ID ProQOL org 78. https://doi.org/ProQOL.org

5. H. S (2010) The ProQOL manual, pp 1-29

6. Tremblay MA, Messervey D, Tremblay M (2011) The job demands-resources model: further evidence for the buffering effect of personal resources. SA J Ind Psychol Tydskr vir Bedryfsielkd 37. https://doi.org/10.4102/sajip.v37i2.876

7. Panagioti M, Geraghty K, Johnson J, Zhou A, Panagopoulou E, Chew-Graham C, Peters D, Hodkinson A, Riley R, Esmail A (2018) Association between physician burnout and patient safety, professionalism, and patient satisfaction: a systematic review and meta-analysis. JAMA Intern Med 178:1317. https://doi.org/10. 1001/JAMAINTERNMED.2018.3713

8. El-bar N, Levy A, Wald HS, Biderman A (2013) Compassion fatigue, burnout and compassion satisfaction among family physicians in the Negev area - a cross-sectional study. Isr J Health Policy Res 2:31. https://doi.org/10.1186/2045-4015-2-31

9. Granek L, Nakash O, Cohen M, Ben-David M, Ariad S (2017) Oncologists' communication about end of life: the relationship among secondary traumatic stress, compassion satisfaction, and approach and avoidance communication. Psychooncology 26: 1980-1986. https://doi.org/10.1002/pon.4289 
10. Medscape National Physician Burnout \& Depression Report 2018. https://www.medscape.com/slideshow/2018-lifestyle-burnoutdepression-6009235\#3. Accessed 8 Oct 2018

11. Simon CE, Pryce JG, Roff LL, Klemmack D (2006) Secondary traumatic stress and oncology social work. J Psychosoc Oncol 23: 1-14. https://doi.org/10.1300/J077v23n04_01

12. Barnes PP, Hospital J, Deshields TL et al (2010) Compassion fatigue and burnout: prevalence among oncology nurses. Clin J Oncol Nurs 14:E56-E62. https://doi.org/10.1188/10.CJON.E56-E62

13. Granek L, Tozer R, Mazzotta P, Ramjaun A, Krzyzanowska M (2012) Nature and impact of grief over patient loss on oncologists' personal and professional lives. Arch Intern Med 172:964-966. https://doi.org/10.1001/archinternmed.2012.1426

14. Giddings $G$ (2010) The ties that bind: a reflection on physician grief. Support Care Cancer 18:1355-1357. https://doi.org/10. 1007/s00520-010-0949-Z

15. Granek L, Ariad S, Shapira S, Bar-Sela G, Ben-David M (2016) Barriers and facilitators in coping with patient death in clinical oncology. Support Care Cancer 24:4219-4227. https://doi.org/10. 1007/s00520-016-3249-4

16. Granek L, Ben-David M, Shapira S, Bar-Sela G, Ariad S (2017) Grief symptoms and difficult patient loss for oncologists in response to patient death. Psychooncology 26:960-966. https://doi. org $/ 10.1002 /$ pon. 4118

17. Granek L, Bartels U, Scheinemann K, Labrecque M, Barrera M (2015) Grief reactions and impact of patient death on pediatric oncologists. Pediatr Blood Cancer 62:134-142. https://doi.org/10. $1002 / \mathrm{pbc} .25228$

18. Shanafelt T, Adjei A, Meyskens FL (2003) When your favorite patient relapses: physician grief and well-being in the practice of oncology. J Clin Oncol 21:2616-2619. https://doi.org/10.1200/ JCO.2003.06.075

19. Lyckholm L (2001) Dealing with stress, burnout, and grief in the practice of oncology. 2:

20. Whippen DA, Canellos GP (1991) Burnout syndrome in the practice of oncology: results of a random survey of 1,000 oncologists. J Clin Oncol 9:1916-1920. https://doi.org/10.1200/JCO.1991.9.10. 1916

21. Magen L, Dekel R (2008) 575-3:555
22. Faschingbauer T (1981) The Texas inventory of grief-revised. Houst Honeycomb Publ

23. Cohen J (1977) Statistical power analysis for the behavioral sciences (rev. ed.). Lawrence Erlbaum Associates. Inc, Hillsdale, NJ, Engl

24. Faul F, Erdfelder E, Lang A-G, Buchner A (2007) G*Power 3: a flexible statistical power analysis program for the social, behavioral, and biomedical sciences. Behav Res Methods 39:175-191

25. Meyer RML, Li A, Klaristenfeld J, Gold JI (2015) Pediatric novice nurses: examining compassion fatigue as a mediator between stress exposure and compassion satisfaction, burnout, and job satisfaction. J Pediatr Nurs 30:174-183. https://doi.org/10.1016/j.pedn.2013.12. 008

26. Braun M, Mikulincer M, Rydall A, Walsh A, Rodin G (2007) Hidden morbidity in cancer: spouse caregivers. J Clin Oncol 25: 4829-4834. https://doi.org/10.1200/JCO.2006.10.0909

27. Haley WE, Levine EG, Brown SL, Bartolucci AA (1987) Stress, appraisal , coping , and social support as predictors of adaptational outcome among dementia caregivers. Psychol Aging 2:323-330

28. Haley WE, Roth DL, Coleton MI, et al (1996) Appraisal, coping, and social support as mediators of well-being in black and white family caregivers of patients with Alzheimer's disease. 64:121-129

29. Bonanno GA, Kaltman S (1999) Toward an integrative perspective on bereavement. 125:760-776

30. Mccann CM, Beddoe E, Mccormick K, et al (2013) Resilience in the health professions : a review of recent literature. $3: 60-81$. https://doi.org/10.5502/ijw.v3i1.4

31. Kulkarni S, Bell H, Hartman JL, Herman-smith RL (2013) Exploring individual and organizational factors contributing to compassion satisfaction, secondary traumatic stress , and burnout in domestic violence service providers. J Soc Soc Work Res 4:114 130. https://doi.org/10.5243/jsswr.2013.8

32. Hunsaker S, Chen H, Maughan D, Heaston S (2015) Factors that influence the development of compassion fatigue, burnout, and compassion satisfaction in emergency department nurses. 186194. https://doi.org/10.1111/jnu.12122

Publisher's note Springer Nature remains neutral with regard to jurisdictional claims in published maps and institutional affiliations. 